\title{
Correlation between microvascular characteristics and the expression of MVD, IGF-1 and STAT3 in the development of colonic polyps carcinogenesis
}

\author{
HONG LIU ${ }^{1}$, JING WU ${ }^{1}$, XIANG-CHUN LIU ${ }^{1}$, NAN WEI ${ }^{1}$, KUI-LIANG LIU ${ }^{1}$, \\ YAN-HUI MA ${ }^{1}, \mathrm{HONG} \mathrm{CHANG}^{2}$ and QUAN ZHOU ${ }^{2}$ \\ Departments of ${ }^{1}$ Gastroenterology and ${ }^{2}$ Pathology, Beijing Shijitan Hospital, \\ Capital Medical University, Beijing 100038, P.R. China
}

Received July 16, 2015; Accepted September 6, 2016

DOI: $10.3892 /$ etm.2016.3927

\begin{abstract}
The aim of the present study was to investigate the correlation between vascular characteristics under narrow band imaging (NBI) and the expression of angiogenic factors of colorectal carcinoma and adenoma, and to evaluate the feasibility of NBI in vivo visualizing angiogenesis. Patients with colorectal polyps, which were pathologically confirmed as early carcinoma and adenoma, were recruited and examined by NBI. The vascular pattern was classified into type I (invisible or faintly visible vasculature), type II (clearly visible microvasculature that is regularly arranged in a round, oval honeycomb-like pattern) and type III (clearly visible microvasculature that is irregularly arranged in size and caliber or has irregular winding). Immunohistochemical staining was performed by cluster of differentiation (CD)34, insulin-like growth factor (IGF)-1 and signal transducer and activator of transcription 3 (STAT3). The histological results were compared with the vascular pattern under NBI. Overall, 64 sites (15 adenocarcinomas, 29 adenomas and 20 normal) from 58 patients were recruited in the study and examined by NBI. A higher proportion of adenomas $(82.1 \%$, $23 / 28)$ and adenocarcinomas $(66.7 \%, 10 / 15)$ had vascular patterns II and III, respectively. The expression of microvessel density (MVD)-CD34 and IGF-1 in normal mucosa compared with adenomas and adenocarcinomas was significantly different ( $\mathrm{P}<0.0001$ and $\mathrm{P}=0.0062$, respectively). MVD-CD34, IGF-1 and STAT3 expression in the sites displayed with vascular patterns I, II, and III was different significantly $(\mathrm{P}<0.0001, \mathrm{P}=0.0010$ and $\mathrm{P}=0.0055$, respectively). The spearman correlation coefficient between NBI vascular pattern and MVD-CD34, IGF-1 and STAT3 expression was $0.67,0.41$ and 0.40 , respectively. In
\end{abstract}

Correspondence to: Dr Jing Wu, Department of Gastroenterology, Beijing Shijitan Hospital, Capital Medical University, 10 Tieyi Road, Beijing 100038, P.R. China

E-mail:wujing_jl@163.com

Key words: narrow band imaging, colorectal adenoma, early colorectal carcinoma, microvascular pattern, angiogenesis conclusion, vascular-pattern analysis and the use of an NBI system may be a promising tool for evaluating angiogenesis of colorectal lesions in real-time endoscopy.

\section{Introduction}

The incidence of colon cancer has significantly increased in China over the past decade, it is now the fourth most common cancer in China $(1,2)$. Clinical evidence supports the belief that the majority of colon cancer arises from precursor lesions and benign adenomatous polyps, that is, the classical adenoma-carcinoma sequence (ACS (3). ACS is a series of events whereby colorectal adenomas develop, initially showing low grade dysplasia, from which some will progress to develop areas of high grade dysplasia and eventually invasive carcinoma. In tumor development, angiogenesis is an important stage, by which solid tumor cells gain access to nutrients and oxygen via an increase in vital blood supply (4). The degree of angiogenesis in malignant tissues is a prognostic factor for colon cancer. Angiogenesis inhibitors offer a novel approach for colon cancer therapy (5).

A previous study demonstrated that carcinoma angiogenesis in cancer occurs at the stage of precancerous lesions (3). Measurement of microvessel density (MVD) is the typical index used to evaluate angiogenesis; insulin-like growth factor 1 (IGF-1), signal transcription factors and signal transducer and activator of transcription 3 (STAT3) can promote angiogenesis (6-8). The present uses endoscopy to improve the observation of angiogenesis, while microvascular morphology on the mucosal surface is observed using narrow band imaging (NBI). The study aimed to observe the expression of MVD, IGF-1 and STAT3 in the process of colonic polyp carcinogenesis and in preneoplastic lesion tissues, and to investigate the correlation of microvascular morphology using NBI. In addition, the current study explored the feasibility of identifying angiogenesis using endoscopic real-time observation.

\section{Materials and methods}

Patient characteristics. All patients presented at the endoscopy room of the Department of Digestion at Beijing Shijitan 
Hospital, Capital Medical University (CMU; Beijing, China) between August 2011 and December 2011. The patients had an average age of 61.5 years (range, 30-89 years), including 36 males and 28 females. All patients were diagnosed with colonic polyps lesions by common endoscopy and NBI, and these were confirmed as colonic early colorectal carcinoma and colonic adenoma by histopathology. The patients with negative colonoscopy results were included and confirmed by polyp biopsy, high frequency electrocutting or surgery. The patients with ulcerative colitis, familial adenomatous polypesis, serrated adenoma and developing colon carcinoma were excluded from the study. Informed consent was obtained from all patients and controls. Written approval for the study was obtained from the Beijing Shijitan Hospital, CMU.

Equipment. The included patients were examined using an NBI endoscope (CF-H260; Olympus Corporation, Tokyo, Japan). The processor type was CV-260SL (Olympus Corporation) with 1280x1024 resolution display, double-band filter of NBI (415 nm and $540 \mathrm{~nm}$ ), structure (A1-A5) and contour emphasis (B1-B5).

Methods. The preparation of NBI endoscope examination was the same to common colonoscopy. The endoscopy reached the ileocecal junction, the terminal intestine and finally the caecum by identification of the appendix, the plica of Y-type cecum and the structures of the ileocecal valve. The endoscope was then drawn out and the colon was observed. Microvascular morphology on the polyp surface was identified according to an NBI model. The lesions were removed by biopsy, endoscopic mucosal resection, endoscopic submucosal dissection or surgery. A sample of biopsy specimen from the boundary between the rectum and the sigmoid colon was obtained from patients with normal colonic mucosa, as the normal mucosa. The specimens were fixed by formaldehyde and embedded by paraffin, then cut into serial sections $(4 \mu \mathrm{m})$. Hematoxylin and eosin staining and histopathological examination were then performed. CD34, IGF-1 and STAT3 (primary antibodies were obtained from Abcam, Cambridge, UK) were detected by immunohistochemical staining according to the specification (9). The histological diagnoses were performed accorded to the criteria of the World Health Organization (10), and was performed separately by two pathologists who were blinded to the endoscopy results.

Immunohistochemical analysis. Weidner (11) criteria for analysis were used. IGF-1 and STAT3 protein staining were analyzed semi-quantitatively. Stained cells were counted under a light microscope (magnification, $\mathrm{x} 400$ ) and the percentage of stained cells was counted in 5 visual fields. The average was then calculated and divided according to the scoring levels: Without positively-stained cells, $0 ;<25 \%$ positively-stained cells, $1 ; 26-50 \%$ positively-stained cells, 2 ; $>50 \%$ positively-stained cells, 3 .

Briefly, IGF-1 and CD34 were used to marker MVD, and a ChemMate EnVision + horseradish peroxidase (HRP)/DAB kit (GK500705; Dako Corp., Troy, MI, USA) was used for immunohistochemical staining. Paraffin-embedded tissue samples were sliced into $4-\mu \mathrm{m}$ sections and heated in the thermostatic oven at $75^{\circ} \mathrm{C}$ for $60 \mathrm{~min}$. Dimethyl benzene was added to the samples to remove paraffin after cooling for $5 \mathrm{~min}$ and the samples were subsequently treated with an alcohol series (100, 90 and 80\% ethanol) for $5 \mathrm{~min}$. Antigen retrieval was performed in a microwave using sodium citrate solution with EDTA solution for $10 \mathrm{~min}$. Slices were washed with PBS three times after cooling for $30 \mathrm{~min} / 2 \mathrm{~min}$, and were incubated with $3 \% \mathrm{H}_{2} \mathrm{O}_{2}$ for $10 \mathrm{~min}$ at room temperature to completely eliminate endogenous peroxidase. Following washing three times with PBS, the samples were incubated with anti-IGF-1 (ab9572) and anti-CD34 (ab81289) primary antibodies (both 1:100; Abcam) for $60 \mathrm{~min}$ in a wet box at $37^{\circ} \mathrm{C}$, and were washed three times with PBS prior to secondary antibody incubation with ChemMate EnVision + HRP for $30 \mathrm{~min}$ at $37^{\circ} \mathrm{C}$. Following washing with PBS thrice, DAB working solution was added to the samples in a wet box. Sterile water was used to wash the samples in order to terminate the reaction prior to counter staining for one minute with hematoxylin. Finally, the slides were dehydrated with an ethanol series for one minute $(75,80$ and $100 \%$ ) and observed under a microscope. Images were captured and analyzed. In the negative control group, PBS was used instead of the primary antibody.

Evaluation of microvesicular morphology by NBI. The microvascular morphology included three types: Type I, no visible microvasculature pattern; type II, microvasculaturearranged along the crypts with an even diameter; and type III, microvasculature irregularly arranged with and uneven diameter (Fig. 1A-C). The microvasculature type of each polyp was recorded under endoscopy and compared with the results of immunohistochemistry, and any correlation between the two was identified.

Statistical analysis. All the data were analyzed by SAS analysis software (version 9.10; SAS Institute, Inc., Cary, NC, USA). All the measurement data were represented as the mean \pm standard deviation. $\mathrm{P}<0.05$ was considered to indicate a statistically significant difference. The differences of the expression levels of MVD, IGF-1 and STAT3 among groups were analyzed by one-way analysis of variance, and multiple comparisons were analyzed by Student-Newman-Keuls. The correlation between endoscopic classification and pathological examination was analyzed by non-parametric Spearman's Rank correlation analysis.

\section{Results}

Patient colon characteristics. In the present study, there were 38 patients with a total of 44 colon lesions and 20 normal colons. A total of 15 colonic early colorectal carcinoma and 29 colonic adenoma were identified in the 44 colon lesions, including 19 low-grade intraepithelial neoplasia, 10 high-grade intraepithelial neoplasia, 14 tubular adenoma and 15 villous adenomas. The histological type and endoscopic type of colorectal lesions are presented in Table I.

Correlation between NBI and histopathological types. According to the NBI types, there were 25 of type I, including 20 of normal positions and 5 of adenomas; 28 of 

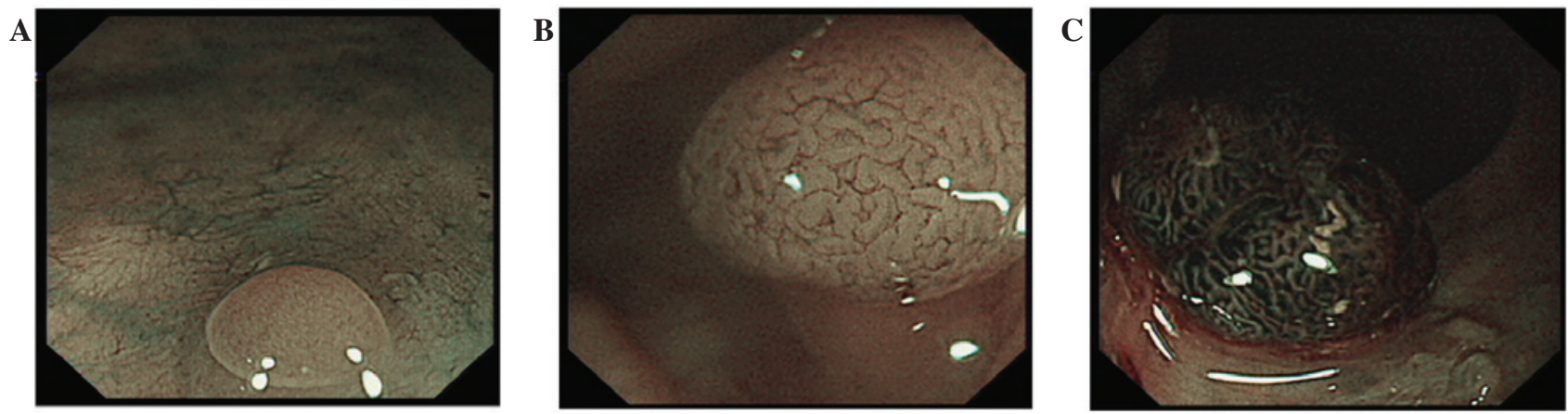

Figure 1. Narrow band imaging microvascular type. (A) Type I, no visible microvascular pattern; (B) type II, microvasculature arranged along the crypts with an even diameter; and (C) type III, microvasculature irregularly arranged with an uneven diameter.

Table I. Histological type and endoscopic type of colorectal lesions.

\begin{tabular}{lc}
\hline Lesions & Colon lesions $(\mathrm{n}=44)$ \\
\hline Average, $\mathrm{cm}$ & 1.44 \\
Early colorectal carcinoma & 1.87 \\
Colonic adenoma & 1.06 \\
Lesion site number & \\
Right colon & 18 \\
Left colon & 26 \\
Morphology number & \\
Eminency type & 23 \\
Superficial type & 19 \\
Depressed type & 2 \\
\hline
\end{tabular}

type II including 23 of adenomas and 5 of early colorectal carcinoma; and 11 of type III including 10 of early colorectal carcinoma and 1 of adenoma. The results indicated that the type II were particularly identified in adenomas with $82.1 \%$ (23/28), while early colorectal carcinoma primarily belonged to type III, with $66.7 \%$ (10/15) (Table II).

Expression of MVD, IGF-1 and STAT3 in early colorectal carcinoma, adenomas and normal mucosa. CD34 was located on membrane and in cytoplasm of vascular endothelial cell. The expression of MVD in normal mucosa, adenomas and early colorectal carcinoma were $8.77 \pm 2.67,21.37 \pm 4.42$ and $21.33 \pm 5.23$, respectively. There were significant differences between the expression of MVD in normal mucosa and adenomas and early colorectal carcinoma $(\mathrm{P}<0.0001)$. There was no significant difference in the expression of MVD between adenomas and early colorectal carcinoma $(P>0.05)$, indicating that MVD increased markedly in adenomas. The expression of IGF-1 in normal mucosa, adenomas and early colorectal carcinoma were $0.50 \pm 0.76,0.59 \pm 0.68$ and $1.27 \pm 0.80$, respectively. There were significant differences in the expression of IGF-1 between the early colorectal carcinoma and normal mucosa and adenoma $(\mathrm{P}=0.0062)$, indicating that IGF-1 increased significantly in lesions. The expression levels of STAT3 in normal mucosa, adenomas
Table II. Correlation of NBI type and histological findings.

\begin{tabular}{lcccc}
\hline $\begin{array}{l}\text { NBI } \\
\text { types }\end{array}$ & Number & Normal & Adenoma & $\begin{array}{c}\text { Early colorectal } \\
\text { carcinoma }\end{array}$ \\
\hline Type I & 25 & 20 & 5 & 0 \\
Type II & 28 & 0 & 23 & 5 \\
Type III & 11 & 0 & 1 & 10 \\
Total & 64 & 20 & 29 & 15 \\
\hline
\end{tabular}

NBI, narrow band imaging.

and early colorectal carcinoma were $0.40 \pm 0.60,0.66 \pm 0.90$ and $1.07 \pm 0.96$, respectively. The expression levels increased gradually among the three, but without significant differences $(\mathrm{P}=0.0713)$. The expression of MVD, IGF-1 and STAT3 in early colorectal carcinoma, adenomas and normal mucosa are presented in Fig. 2 and Table III.

Correlation between microvascular characteristics and $M V D$, and the expression of IGF-1 and STAT3. In NBI types, there were 25 cases of type I expressing MVD (10.85 \pm 5.20$)$, 28 cases of type II expressing MVD (21.46 \pm 4.21$)$ and 11 cases of type III expressing $(22.09 \pm 5.84)$. There were significant differences among the three $(\mathrm{P}<0.0001)$. The IGF-1 expression in type I $(0.44 \pm 0.71)$, type II $(0.68 \pm 0.67)$ and type III $(1.45 \pm 0.82)$ were significantly different from each other $(\mathrm{P}=0.0010)$. The STAT3 expression in type I $(0.32 \pm 0.57)$, type II $(0.75 \pm 0.93)$ and type III $(1.27 \pm 0.90)$ were significantly different from each other $(\mathrm{P}=0.0055$; Table IV).

By analyzing the correlation between NBI types and MVD expression, the results of the present study demonstrated that Spearman's correlation coefficient was 0.67, indicating a correlation between the two. Under endoscopy, as the vessel number increased the color became deeper, and the expression of MVD increased gradually. The correlation coefficients between type I, type II and type III and the expression of IGF-1 and STAT3 were 0.41 and 0.40 , respectively, indicating that the vascular morphology was poorly correlated with the expression of IGF-1 and STAT3. The vascular morphology under endoscopy did not reflect the expression of IGF-1 and STAT3. 
Table III. Expression of MVD, IGF-1 and STAT3 in early colorectal carcinoma, adenoma and normal mucosa.

\begin{tabular}{lccccccr}
\hline Histology & Cases & MVD & P-value & IGF-1 & P-value & STAT3 & P-value \\
\hline Normal mucosa & 20 & $8.77 \pm 2.67$ & & $0.50 \pm 0.76$ & & $0.40 \pm 0.60$ & \\
Colonic adenoma & 29 & $21.37 \pm 4.42$ & $<0.0001$ & $0.59 \pm 0.68$ & 0.0062 & $0.66 \pm 0.90$ & 0.0713 \\
Early colorectal carcinoma & 15 & $21.33 \pm 5.23$ & & $1.27 \pm 0.80$ & & $1.07 \pm 0.96$ &
\end{tabular}

MVD, microvessel density; IGF-1, insulin-like growth factor 1; STAT3, signal transducer and activator of transcription 3.

Table IV. Correlation of NBI type and expression of MVD, IGF-1 and STAT3.

\begin{tabular}{lcccccc}
\hline NBI & Cases & MVD & P-value & IGF-1 & P-value & STAT3 \\
\hline Type I & 25 & $10.85 \pm 5.20$ & & $0.44 \pm 0.71$ & & $0.32 \pm 0.57$ \\
Type II & 28 & $21.46 \pm 4.21$ & $<0.0001$ & $0.68 \pm 0.67$ & 0.0010 & $0.75 \pm 0.93$ \\
Type III & 11 & $22.09 \pm 5.84$ & & $1.45 \pm 0.82$ & & $1.27 \pm 0.90$ \\
\hline
\end{tabular}

NBI, narrow band imaging; MVD, microvessel density; IGF-1, insulin-like growth factor 1; STAT3, signal transducer and activator of transcription 3 .
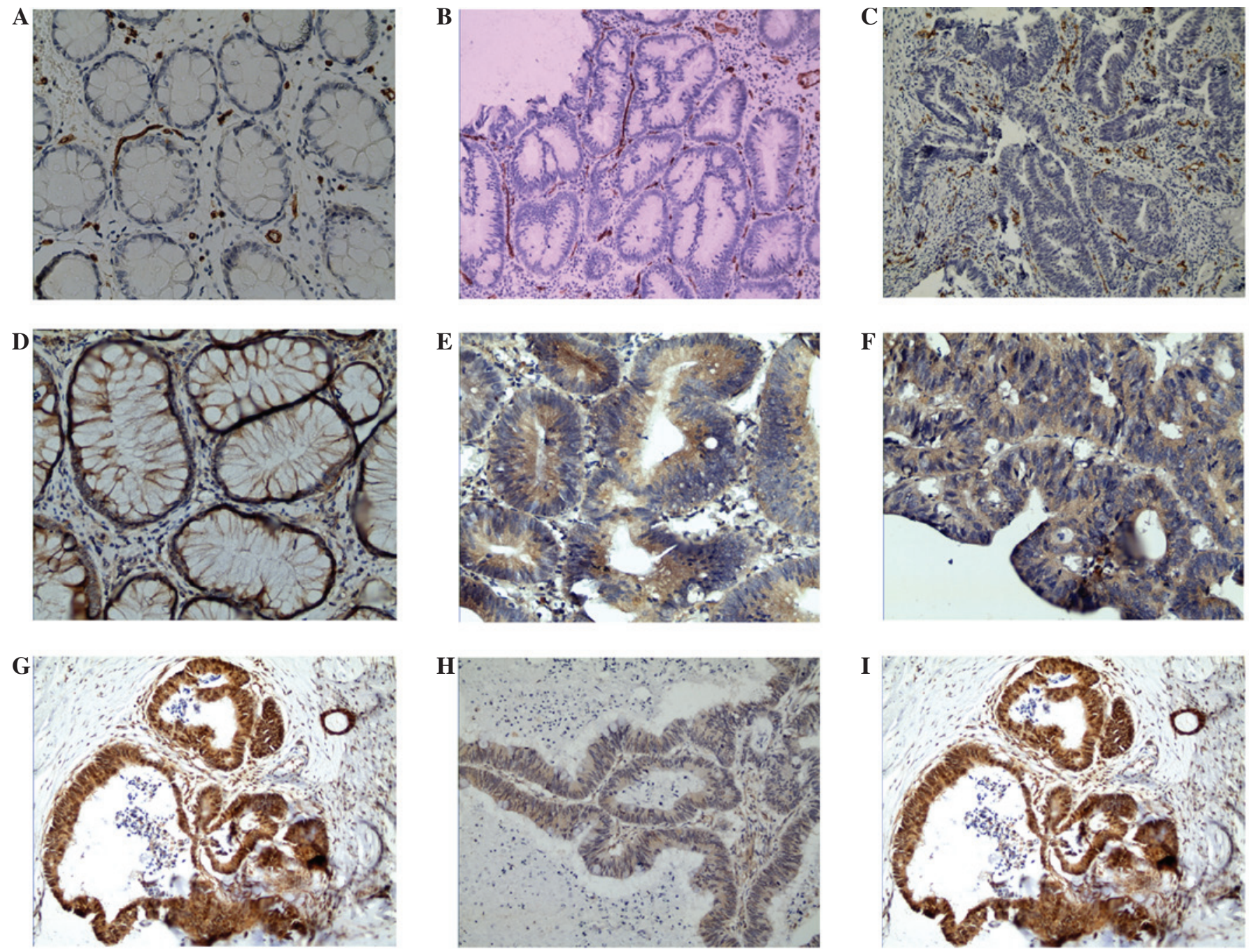

Figure 2. Expression of CD34, IGF-1 and STAT3 in colorectal cancer, adenoma and normal mucosa. The expression of (A) CD34 in normal mucosa (magnification, x400), (B) CD34 in adenoma (magnification, x200), (C) CD34 in colorectal cancer (magnification, x200), (D) IGF-1 in normal mucosa (x400), (E) IGF-1 in adenoma (x400), (F) IGF-1 in colorectal cancer (x400), (G) STAT3 in normal mucosa (x200), (H) STAT3 in adenoma (x200) and (I) STAT3 in colorectal cancer (x200). CD, cluster of differentiation; IGF-1, insulin-like growth factor-1; STAT3, signal transducer and activator of transcription 3. 


\section{Discussion}

Angiogenesis is important in the diagnosis and treatment of tumors. A number of studies have indicated that angiogenesis increases at the stage of precancerous lesions. NBI endoscopy, as a novel technology, enables the observation of angiogenesis in vivo $(3,12)$. In the present study, the microvascular morphology changes of colonic polyps was observed to be positively correlated with angiogenesis indexes in histological examination under NBI endoscopy. As the number of microvessels increased and the color deepened, the angiogenesis factor expression increased in the tissues, which indicated the feasibility of observing angiogenesis under endoscopy.

There was a correlation between the endoscopic classification and histological results, which is consistent with a previous study (13). Type I (no visible microvascular pattern) indicated normal colonic mucosa and hyperplastic polyps, while type II (microvasculature arranged along the crypts with an even diameter) demonstrated that there was no correlation between microvascular morphology and colonic adenoma (14,15). Type III (irregularly arranged microvasculature with an uneven diameter) indicated early colorectal carcinoma. The results mentioned above are all consistent with previous studies (16-18).

In the present study, the expression of MVD was examined by labeling vascular endothelial with CD34 by immunohistochemistry. The results indicated that MVD in colonic adenoma and early colorectal carcinoma was higher than in normal colonic mucosa, and that MVD increased markedly in adenoma. Previous research has demonstrated that the increase of MVD depends on the expression level of angiogenesis factors (19). IGF-1 is a type of somatomedin, which can promote tumor angiogenesis (20), while STAT3 is an important meeting point in numerous signal transduction pathways of angiogenesis $(8,21)$. The current study indicated that there was a similar tendency between MVD and the expression of IGF-1 and STAT3, and IGF-1 and STAT3 increased gradually in normal mucosa, adenomas and early colorectal carcinoma. IGF-1 was without significant increase in adenomas, but increased markedly in early colorectal carcinoma, which indicates that the tendency of increasing IGF-1 in normal mucosa, adenomas and early colorectal carcinoma is different from MVD. MVD increasing may be caused by the other pro-angiogenic factors.

In the current study on the correlation between microvascular morphology and angiogenesis indexes under endoscopy, there were significant differences in the expression of MVD, IGF-1 and STAT3 in NBI types, including type I, type II and type III. The correlation coefficient between NBI types and MVD was 0.67 , which indicated a correlation between the two. As the number of microvessels increased and the color deepened, the MVD increased. The correlation coefficients between type I, type II and type III, and the expression of IGF-1 and STAT3, were 0.41 and 0.40 , respectively, which indicated that the vascular morphology was poorly correlated to the expression of IGF-1 and STAT3. Vascular morphology under endoscopy did not reflect the expression of IGF-1 and STAT3. Together, the results of the present study demonstrate that the vascular morphology observed under endoscopy may reflect MVD, but with poor correlation to the expression of IGF-1, STAT3 and other pro-angiogenic factors.
In the present study, consecutive patients were not chosen as study subjects, because in conventional endoscopic examination there were a large number of hyperplastic polyps and low-grade tubular polyps. In order to observe the relationship between microvessel and histological change in carcinogenesis, adenomas were chosen as targets, particularly high-grade polys. The microvascular morphological changes were poorly correlated to the pro-angiogenic factors, which indicated that other factors should be investigated in a bigger sample size in future investigations.

In conclusion, NBI endoscopic real-time observation is a promising examining method for the evaluation of tissue angiogenesis, which indicates the feasibility of observing angiogenesis by endoscopy.

\section{Acknowledgements}

The present study was funded by Funding For Training Talents in Beijing City (grant no. 2011D003034000009) and Beijing City Health System '215' High Levels of Health Technical Personnel Training Aid (grant no. 2014-3-047).

\section{References}

1. Chen Q, Liu ZC and Cheng LP: Analysis of incidence and mortality of colorectal cancer in china, 2003-2007. Chin Cancer 21: 179-182, 2012.

2. Park HM, Woo H, Jung SJ, Jung KW, Shin HR, Shin A: Colorectal cancer incidence in 5 Asian countries by subsite: An analysis of cancer incidence in five continents (1998-2007). Cancer Epidemiology 45: 65-70, 2016.

3. Staton CA, Chetwood AS, Cameron IC, Cross SS, Brown NJ and Reed MW: The angiogenic switch occurs at the adenoma stage of the adenoma carcinoma sequence in colorectal cancer. Gut 56: 1426-1432, 2007.

4. Sharma S, Sharma MC and Sarkar C: Morphology of angiogenesis in human cancer: a conceptual overview, histoprognostic perspective and significance of neoangiogenesis. Histopathol 46: 481-489. 2005.

5. Gacche RN and Meshram RJ: Angiogenic factors as potential drug target: Efficacy and limitations of anti-angiogenic therapy. Biochimica et Biophysica Acta 1846: 161-179, 2014

6. Ren J, Jin W, Gao YE, Zhang Y, Zhang X, Zhao D, Ma H, Li Z, Wang J, Xiao L, et al: Relations between GPR4 expression, microvascular density (MVD) and clinical pathological characteristics of patients with epithelial ovarian carcinoma (EOC). Curr Pharm Des 20: 1904-1916, 2014.

7. Jacobo SM and Kazlauskas A: Insulin-like growth factor 1 (IGF-1) stabilizes nascent blood vessels. J Biol Chem 290: 6349-6360, 2015.

8. Zhu Y, Xu Y, Chen D, Zhang C, Rui W, Zhao J, Zhu Q, Wu Y, Shen Z, Wang W, et al: Expression of STAT3 and IGF2 in adrenocortical carcinoma and its relationship with angiogenesis. Clin Transl Oncol 16: 644-649, 2014.

9. Qian WF, Guan WX and Gao Y: The expression of STAT3 in colorectal carcinoma and its effects on angiogenesis. Zhong Guo Lin Chuang Jie Po Xue Za Zhi 27: 310-316, 2009 (In Chinese).

10. Sengiz S, Pabuççuoğlu U and Sarioğlu S: Immunohistological comparison of the World Health Organization (WHO) and Ljubljana classifications on the grading of preneoplastic lesions of the larynx. Pathol Res Pract 200: 181-188, 2004.

11. Weidner N: Tumor angiogenesis: Review of current applications in tumor prognostication. Semin Diagn Pathol 10: 302-313, 1993.

12. Möbius C, Stein HJ, Becker I, Feith M, Theisen J, Gais P, Jütting U and Siewert JR: The 'angiogenic switch' in the progression from Barrett's metaplasia to esophageal adenocarcinoma. Eur J Surg Oncol 29: 890-894, 2003.

13. Liu H, Wu J, Lin XC, Gao BX, Jiang GJ, Wei N and Wang CH: Narrow band imaging without magnification for differential diagnosis of colorectal adenoma and hyperplastic polyps. Zhong Hua Xiao Hua Za Zhi 31: 798-802, 2011 (In Chinese).

14. Rastogi A: Optical diagnosis of small colorectal polyp histology with high-definition colonoscopy using narrow band imaging. Clin Endosc 46: 120-129, 2013. 
15. Goto N, Kusaka T, Tomita Y, Tanaka H, Itokawa Y, Koshikawa Y, Yamaguchi D, Nakai Y, Fujii S and Kokuryu H: Magnifying narrow-band imaging with acetic acid to diagnose early colorectal cancer. World J Gastroenterol 20: 16306-16310, 2014.

16. Hayashi N, Tanaka S, Hewett DG, Kaltenbach TR, Sano Y, Ponchon T, Saunders BP, Rex DK and Soetikno RM: Endoscopic prediction of deep submucosal invasive carcinoma: Validation of the narrow-band imaging international colorectal endoscopic (NICE) classification. Gastrointest Endosc 78: 625-632, 2013.

17. Rastogi A: Optical diagnosis of small colorectal polyp histology with high-definition colonoscopy usingnarrow band imaging. Clin Endosc 46: 120-129, 2013.
18. Hayashi N, Tanaka S, Kanao H, Oka S, Yoshida S and Chayama K: Relationship between narrow-band imaging magnifying observation and pit pattern diagnosis in colorectal tumors. Digestion 87: 53-58, 2013.

19. Liu H, Xu KX, Cao LJ, Wang H and Kang T: Expression and biological significance of Leptin, Leptin receptor, VEGF and CD34 in colorectal carcinoma. Zhong Guo Zhong Liu Lin Chuang 2009, 36: 934-936, 2009 (In Chinese).

20. Han Z, Tian X, Tan J, Long YH and Wang H: Correlation between serum IGF-1 and VEGF expression in gastric cancer. Wuhan Da Xue Xue Bao 35: 525-527, 2014.

21. Chen Z and Han ZC: STAT3: A critical transcription activator in angiogenesis. Med Res Rev 28: 185-200, 2008. 